\title{
Downregulation of MCM8 expression restrains the malignant progression of cholangiocarcinoma
}

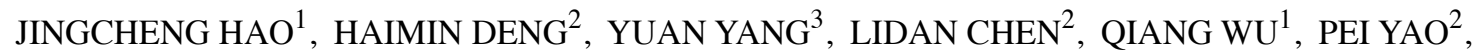 \\ JUNEN $\mathrm{LI}^{2}$, BOWEN LI ${ }^{2}$, XUELI JIN ${ }^{1}$, HAIQING WANG ${ }^{4}$ and HUAXIN DUAN ${ }^{2,5,6}$
}

\begin{abstract}
${ }^{1}$ Department of Hepatobiliary and Vascular Surgery, Clinical Medical College and The First Affiliated Hospital of Chengdu Medical College, Chengdu, Sichuan 610500; ${ }^{2}$ Department of Oncology, Hunan Provincial People's Hospital, The First Affiliated Hospital of Hunan Normal University, Changsha, Hunan 410005; ${ }^{3}$ Department of Rheumatology and

Immunology, Clinical Medical College and The First Affiliated Hospital of Chengdu Medical College,

Chengdu, Sichuan 610500; ${ }^{4}$ Department of Hepatopancreatobiliary Surgery, Sichuan Cancer Hospital and Institute,

Sichuan Cancer Center, School of Medicine, University of Electronic Science and Technology of China,

Chengdu, Sichuan 610041; ${ }^{5}$ Key laboratory of Study and Discovery of Small Targeted Molecules of Hunan Province,

Hunan Normal University, Changsha, Hunan 410013; ${ }^{6}$ Laboratory of Oncology, Institute of Translational Medicine,

Hunan Provincial People's Hospital, Changsha, Hunan 410005, P.R. China
\end{abstract}

Received March 5, 2021; Accepted August 10, 2021

DOI: $10.3892 /$ or.2021.8186

\begin{abstract}
Cholangiocarcinoma (CCA) is a highly aggressive malignant tumor with an extremely poor prognosis. Minichromosome maintenance 8 homologous recombination repair factor (MCM8) is a helicase involved in the elongation step of DNA replication and tumorigenesis. In the present study, the clinical significance and biological function of MCM8 in CCA were investigated. The expression levels of MCM8 in CCA and paracancerous tissues were analyzed using immunohistochemical staining. The potential mechanisms underlying MCM8 and the biological effects of MCM8 in CCA cells were explored using in vitro assays and in vivo mouse xenograft models. The high expression levels of MCM8 in CCA has important clinical significance in predicting disease progression. Knockdown of MCM8 decreased proliferation, promoted apoptosis and suppressed migration of CCA cells. MCM8 knockdown also suppressed tumor growth in vivo. Mechanistically, MCM8 knockdown led to the abnormal downregulation of survivin, XIAP, HSP27, IGF-1sR, sTNF-R1, sTNF-R2, TNF- $\alpha$ and TNF- $\beta$. Furthermore, downregulation of MCM8 expression inhibited the PI3K/Akt signaling pathway and induced the MAPK9 signaling pathway. MCM8 promoted the malignant progression of CCA, indicating that inhibition
\end{abstract}

Correspondence to: Dr Huaxin Duan, Department of Oncology, Hunan Provincial People's Hospital, The First Affiliated Hospital of Hunan Normal University, No. 61 Jiefang West Road, Changsha, Hunan 410005, P.R. China

E-mail: 317102912@qq.com

Key words: cholangiocarcinoma, minichromosome maintenance 8 homologous recombination repair factor, proliferation, apoptosis, migration of MCM8 may have the potential to serve as a novel molecular targeted therapy.

\section{Introduction}

Cholangiocarcinoma (CCA) is a term used to describe a cluster of highly heterogeneous malignant tumors that arise in any part of the biliary tree (1). CCA is a highly malignant, relatively rare disease, yet the incidence of CCA is increasing worldwide (2). At present, the main treatment strategies of CCA include surgical resection, liver transplantation, systemic chemotherapy and local treatment (3). In addition, CCA often remains concealed, is highly heterogeneous, highly invasive and has a high tolerance to chemotherapy. These factors have a detrimental impact on the effectiveness of existing treatments which leads to high rates of mortality (4). Thus, the complexity of the tumor requires further investigation to allow the development of innovative therapies to improve patient outcomes. Currently, candidate molecular targets for precision medicine are being identified and evaluated in clinical trials (5). The molecular mechanisms underlying CCA require in depth investigation to identify key small molecular targets and associated signaling pathways that may serve as novel therapeutic approaches $(5,6)$. The minichromosome maintenance (MCM) protein family was initially discovered in the budding yeast Saccharomyces cerevisiae and received extensive attention (7). Initial study has revealed that the MCM family plays a key role in DNA replication (8). In previous studies, it has been reported that MCM2-7 are overexpressed in esophageal squamous cell carcinoma, cervical, gastric and breast cancer as well as other cancers (9-12). Minichromosome maintenance 8 homologous recombination repair factor (MCM8) and the related MCM9 were recently discovered as members of the MCM family. MCM8 has significant homology with MCM7, and is a helicase involved in the elongation step of DNA 
replication (13). The findings of a recent study indicated that MCM2-7, MCM8 and MCM10 are overexpressed in hepatocellular carcinoma (HCC) (14). However, the specific molecular mechanism underlying the role of MCM8 in CCA has yet to be elucidated.

In the present study, the clinical significance and biological function of MCM8 in CCA progression were investigated. The expression of MCM8 in CCA and the effects of MCM8 knockdown on the proliferation, apoptosis and migration of CCA cells were examined. It was also investigated whether the effects of MCM8 knockdown were mediated via regulation of apoptosis-related protein expression. The aim was to elucidate whether MCM8 knockdown exerts an inhibitory effect on CCA and determine its therapeutic potential as a molecular target.

\section{Materials and methods}

Tissue collection and immunohistochemical (IHC) staining. A total of 122 human CCA and paired paracancerous tissue samples were purchased from Shanghai Outdo Biotech Co., Ltd. (cat. no. JS W-11-01; http://www.superchip.com.cn). All tissues were from patients with CCA who underwent surgery, and none of the patients received any chemotherapy or radiation before surgery. IHC staining was performed as previously described in the literature (15). The experimental protocols and the human tissues used were approved (approval no. 20200305) by the Ethics Committee of Clinical Research of The First Affiliated Hospital of Chengdu Medical College (Chengdu, China). Formalin-fixed and paraffin-embedded tissues $(4 \mu \mathrm{m})$ were deparaffinized in xylene and rehydrated in ethanol. Subsequently, the tissues were incubated with MCM8 primary antibody (1:100; cat. no. PA5-41325; Invitrogen; Thermo Fisher Scientific, Inc.) at $4^{\circ} \mathrm{C}$ for $4 \mathrm{~h}$ and an HRP-conjugated secondary antibody (1:400; cat. no. ab6721; Abcam) at room temperature for $1 \mathrm{~h}$. Following incubation, the tissues were stained with 3,3'-diaminobenzidine solution and hematoxylin at room temperature for $10 \mathrm{~min}$. IHC scores were determined by staining percentage and staining intensity as previously described by Haonon et al (15). In addition, the median IHC score was used to verify the expression levels of MCM8 in CCA.

Cell culture. The human CCA cell lines HCCC-9810, RBE and HuCCT1 were obtained from the Cell Bank of Type Culture Collection of The Chinese Academy of Sciences and cultured at $37^{\circ} \mathrm{C}$ in RPMI-1640 medium supplemented with $10 \% \mathrm{FBS}$, $100 \mathrm{mg} / \mathrm{ml}$ streptomycin and $100 \mathrm{IU} / \mathrm{ml}$ penicillin (all from Gibco; Thermo Fisher Scientific, Inc.) in a humidified atmosphere of $5 \% \mathrm{CO}_{2}$.

Lentivirus production and cell transduction. A total of three short hairpin (sh)RNAs specifically targeting MCM8 (shMCM8) were designed for MCM8 knockdown and the sequences were as follows: RNAi-1: 5'-TGGCAATACATC AGGTGTTAA-3'; RNAi-2: 5'-CTGGAATTGTCAAAG TCTCAA-3'; and RNAi-3: 5'-AGGCAGCTGGAATCTTTG ATT-3'. The negative control was a scrambled short interfering (si)RNA (shCtrl: 5'-CCG GAGGGATTGACTTAGAGCAAA TCTCGAGATTTGCTCTAAGTCAATCCCTTTTTTG-3'). Lentivirus vectors BR-V108 were purchased from Yi Berry
Biological Medicine Technology Co., LTD (http://www. bioscienceres.com). and labeled with green fluorescent protein (GFP) and resistance tag (puromycin, $400 \mathrm{ng} / \mathrm{ml}$ ) for cell screening. Human CCA cell lines HCCC-9810 and RBE were cultured in 6 -well plates $\left(2 \times 10^{5}\right.$ cells/well; Corning, Inc.) at $37^{\circ} \mathrm{C}$ for $24 \mathrm{~h}$ for second-generation lentiviral transduction. Recombinant lentivirus particles $\left(1 \times 10^{7} \mathrm{TU} / \mathrm{ml}\right)$ contained in shMCM8 or shCtrl sequences were used to transduce CCA cell lines HCCC-9810 and RBE $\left(5 \times 10^{5}\right.$ cells $\left./ \mathrm{ml}\right)$ using Lipofectamine $^{\circledR} 3000$ (Invitrogen; Thermo Fisher Scientific, Inc.) in RPMI-1640 medium with $10 \% \mathrm{FBS}$ at a multiplicity of infection of 10 for $40 \mathrm{~min}$ at $37^{\circ} \mathrm{C}$. A total of $72 \mathrm{~h}$ after transduction, the expression of GFP in the cells was observed under a fluorescence microscope (magnification, x200; Olympus Corporation), and the cells were cultured for $24 \mathrm{~h}$ for subsequent experiments.

$R N A$ extraction and reverse transcription-quantitative (RT- $q)$ $P C R$. RNA was extracted using TRIzol reagent (Invitrogen; Thermo Fisher Scientific, Inc.) from target cells. Total RNA was reverse transcribed into cDNA using the M-MLV Reverse Transcriptase kit (Promega Corporation), according to the manufacturer's protocol. qPCR was subsequently performed as follows: Initial denaturation at $95^{\circ} \mathrm{C}$ for $15 \mathrm{~min}$; 40 of cycles of denaturation, annealing and elongation at $55^{\circ} \mathrm{C}$ for $15 \mathrm{~min}$; and a final extension at $85^{\circ} \mathrm{C}$ for 2 min using SYBR-Green Master Mix Kit (Vazyme Biotech Co., Ltd.). The relative mRNA expression of MCM8 was quantified with cycle threshold $(\mathrm{Ct})$ values and normalized using the $2^{-\Delta \Delta \mathrm{Cq}}$ method (16). The primer sequences used were as follows: MCM8 upstream, 5'-ATGGCTTTTCTTTGTGCTGC-3' and downstream, 5'-CCAGTCCATCGTAACTGTGAGA-3'; GAPDH (internal control) upstream, 5'-TGACTTCAACAGCGACACCCA-3' and downstream, 5'-CACCCTGTTGCTGTAGCCAAA-3'.

Western blotting. Total protein obtained using RIPA lysis buffer (Beyotime, Jiangsu, China) from HCCC-9810 and RBE cells and quantified using a BCA Protein Assay Kit (Pierce; Thermo Fisher Scientific, Inc.) and $20 \mu \mathrm{g} /$ lane protein was separated by SDS-PAGE on a $10 \%$ gel [30\% acrylamide/bis-acrylamide; $1.5 \mathrm{~mol} / 1$ Tris ( $\mathrm{pH} 8.8$ ); $10 \%$ SDS; $10 \%$ ammonium persulfate and tetramethylethylenediamine]. The separated proteins were transferred onto PVDF membranes and blocked using TBST solution containing 5\% skimmed milk and $0.5 \mathrm{ml}$ Tween-20 at $4^{\circ} \mathrm{C}$ for $1 \mathrm{~h}$. Immunostaining was performed using target primary antibodies: Anti-MCM8 (1:1,000; cat. no. PA5-41325; Invitrogen), anti-Akt, (1:1,000; product no. 4685; CST), anti-p-Akt (1:1,000; cat. no. BS-5193R; Bioss), anti-MAPK9 (1:1,000; product code ab76125; Abcam), anti-PIK3CA (1:1,000; product code ab40776; Abcam), GAPDH (1:3,000; cat. no. AP0063; Bioworld) at $4{ }^{\circ} \mathrm{C}$ for $4 \mathrm{~h}$ and corresponding secondary antibody goat anti-rabbit (1:3,000; cat. no. A0208; Beyotime Institute of Biotechnology) at room temperature for $2 \mathrm{~h}$. Protein bands were visualized using the Amersham ECL + TM Western Blot system (Cytiva) and signal intensities were analyzed using ImageJ software version 1.8.0.112 (National Institutes of Health).

MTT assay. After 5 days of transduction, HCCC-9810 and RBE cells were inoculated overnight on 6-well plates at 
2,000 cells/well. A total of $20 \mu \mathrm{l}$ of $5 \mathrm{mg} / \mathrm{ml}$ MTT solution (cat. no. JT343; Genview) was added to each well for $4 \mathrm{~h}$. Subsequently, $100 \mathrm{ml} /$ well DMSO was added and OD values at $490 \mathrm{~nm}$ were obtained at 24,48, 72, 96 and $120 \mathrm{~h}$ using a microplate reader. The cell viability was calculated as a ratio of the OD of treated cells to the OD of control cells.

Cell colony formation assay. After 5 days of transduction, HCCC-9810 and RBE cells were inoculated on 6-well plates at a density of 1,000 cells/well for 8 days. The number of cells in a single clone was greater than 50 , and the size between 0.3-1.0 mm. Subsequently, the cells were fixed with $1 \mathrm{ml} 4 \%$ paraformaldehyde at room temperature for 30-60 min and stained with $500 \mu \mathrm{l}$ Giemsa for 10-20 min at room temperature. After washing the cells several times with double distilled water, the cells were captured and counted with a camera (Sony Group Corporation).

Cell apoptosis detection by flow cytometry. After 5 days of transduction, HCCC-9810 and RBE cells were inoculated on 6-well plates at a seeding density of 1,000 cells/well for 10 days. The cells were washed with PBS, centrifuged $\left(4^{\circ} \mathrm{C}\right.$, $12,000 \times \mathrm{g}, 1 \mathrm{~min}$ ) and resuspended. Subsequently, $500 \mu \mathrm{l}$ of diluted 1X Annexin V binding buffer (cat. no. 88-8007-74; eBioscience; Thermo Fisher Scientific, Inc.) was added and cells were stained with $10 \mu \mathrm{l}$ Annexin V-APC for $10-15 \mathrm{~min}$ at room temperature in the dark. Subsequently, $5 \mu 1$ of $50 \mu \mathrm{g} / \mathrm{ml}$ PI was used for staining the cells at room temperature for $20 \mathrm{~min}$ and $1 \mathrm{X}$ binding buffer was added to assess cell apoptosis using the Guava easyCyte 12HT Benchtop Flow Cytometer and GuavaSuite 19.0 Software (Java; both from Luminex Corporation).

Human apoptosis antibody array. Prepared target cell protein was used to investigate the expression levels of apoptosis-related proteins using the human apoptosis antibody array (cat. no. ab134001; Abcam). After the membrane was blocked with array buffer at room temperature for $1 \mathrm{~h}$, prepared HuCCT1 cell protein was incubated with the membrane at $4^{\circ} \mathrm{C}$ overnight. The array was subsequently washed, and incubated with detection antibodies (1:100; cat. no. ab134001; Abcam) and streptavidin-HRP (cat. no. ab134001; Abcam) at room temperature for $1 \mathrm{~h}$. Protein expression was visualized using the Amersham ECL + TM Western Blot system (Cytiva) and signal intensities were analyzed using ImageJ software version 1.8.0.112.

Wound-healing assay. After 5 days of transduction, HCCC-9810 and RBE cells were inoculated on 6-well plates at a seeding density of $5 \times 10^{4}$ cells/well. When the cells had reached a confluence of $>90 \%$, the culture medium was replaced with fresh medium. The cell layer was scratched using the scratch instrument (cat. no. VP408FH; V\&P Scientific, Inc.) and the cells were cultured in serum-free medium. The shadow area in the center of the 6-well plate was used as a reference and the scratch was in the center of the image. According to the preliminary experiments, images were captured at $0,4,8$, 24 and $48 \mathrm{~h}$ using a fluorescence microscope (magnification, x200; Olympus Corporation) and the cell migration rate of each group was calculated.
Transwell assay. A total of five days after transduction, HCCC-9810 and RBE cells were seeded on a 24-well plate $\left(5 \times 10^{4}\right.$ cells/well) for $24 \mathrm{~h}$. The chamber in the Transwell transfer test kit (Corning, Inc.) was composed of 24-well tissue culture plates and 12-well cell culture inserts. A total of $100 \mu \mathrm{l}$ of the cell suspension $\left(5 \times 10^{5}\right.$ cells) was loaded into the upper chamber of the Transwell and the lower chamber of the Transwell was filled with $500 \mu \mathrm{l}$ culture medium containing $30 \% \mathrm{FBS}$ for incubation at $37^{\circ} \mathrm{C}$ for $48 \mathrm{~h}$. The insert contained a polycarbonate membrane with a pore size of $8 \mu \mathrm{m}$, and the cells migrated and adhered to the bottom of the polycarbonate membrane. The cells were fixed with $4 \%$ paraformaldehyde for $30 \mathrm{~min}$ and stained with $0.1 \%$ crystal violet solution at room temperature for $20 \mathrm{~min}$. The non-migrating cells were removed and migrated cells were stained. Following washing with PBS, 5 fields of view per well were selected randomly under a fluorescence microscope (magnification, x200; Olympus Corporation), and images were captured for enumeration of the cells.

Mouse xenograft model. Mouse experiments were approved by the Ethics Committee of Chengdu Medical College (approval no. 20190213), following the Guidelines and Procedures for Animal Care and Protection (http://www. lascn.net/Item/7281.aspx). A total of 4-week-old female BALB/c nude mice (Shanghai Lingchang Biotechnology Co., Ltd.) with a body weight of 15-25 g were used to construct the pathogen-free xenograft model. Mice were kept in a non-toxic metal cage with a 12-h light/dark cycle, a constant temperature of $20-22^{\circ} \mathrm{C}$, humidity at $30-70 \%$, ventilation every 10-20 times/h and food (cat. no. 000521; Shanghai ZeYa; http://www.shzeya.com) and water intake at any time. At 5 days after transduction, $0.2 \mathrm{ml}$ of shMCM8 and shCtrl HuCCT1 suspended with PBS with a cell concentration of $4 \times 10^{6}$ cells $/ \mathrm{ml}$ were injected into the left back of each mouse. The weight and volume of tumors was monitored twice a week, starting at 10 days following injection (calculated by $\pi / 6 \times \mathrm{L} \mathrm{x} \mathrm{W}^{2}$; where $\mathrm{L}$ was the long diameter and $\mathrm{W}$ was the short diameter). At 46 days after injection, mice were anesthetized using $0.7 \%$ pentobarbital sodium at a dose of $40 \mathrm{mg} / \mathrm{kg}$. Subsequently, fluorescence was observed under the imaging system (emission wavelength $510 \mathrm{~nm}$; IVIS Spectrum; Perkin Elmer). Mice were sacrificed by intraperitoneal injection of sodium pentobarbital at a dose of $100 \mathrm{mg} / \mathrm{kg}$ according to the American Veterinary Medical Association guidelines (http://www.lascn.net/Item/91107.aspx). The tumors were removed, weighed and images were captured and tumor slides were subjected to Ki67 expression detection using the IHC staining method. Tumor specimens of shMCM8 and shCtrl $(4 \mu \mathrm{m})$ were fixed with formalin for $30 \mathrm{~min}$, dewaxed with xylene for $15 \mathrm{~min}$ and rehydrated with $100 \%$ alcohol for $10 \mathrm{~min}$. $\mathrm{PBS}-\mathrm{H}_{2} \mathrm{O}_{2}$ with $0.1 \%$ Tween-20 were added and the tissues were washed three times with PBS, 5 min each time. Citric acid buffer was added for antigen retrieval, with heating at $120^{\circ} \mathrm{C}$ for $20 \mathrm{~min}$. The tumor tissues were incubated with the anti-Ki67 (1:200; cat. no. ab16667, Abcam) at $4^{\circ} \mathrm{C}$ overnight and secondary antibody incubation with HRP goat anti-rabbit IgG (1:400; cat. no. ab6721; Abcam) at room temperature for $2 \mathrm{~h}$. Images were collected with a microscope (magnification, x200; Olympus Corporation). 
Table I. Expression patterns in cholangiocarcinoma tissues and para-carcinoma tissues revealed by immunohistochemical analysis.

\begin{tabular}{lcccccc}
\hline & \multicolumn{2}{c}{ Tumor tissue } & & \multicolumn{2}{c}{ Para-carcinoma tissue } & \\
\cline { 2 - 3 } MCM8 expression & Cases & Percentage (\%) & & Cases & Percentage (\%) & P-value \\
\hline Low & 47 & 52.8 & & 23 & 74.2 & $4.098 \times 10^{-6}$ \\
High & 42 & 47.2 & & 8 & 25.8 & \\
\hline
\end{tabular}

A
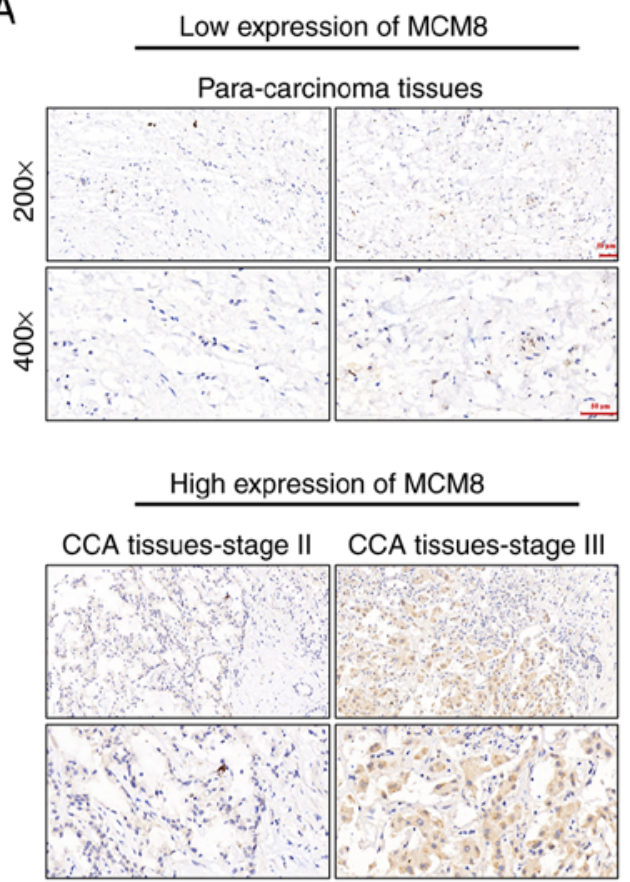

B

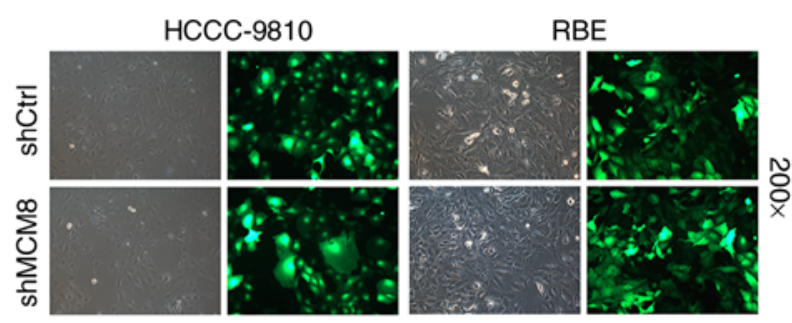

C

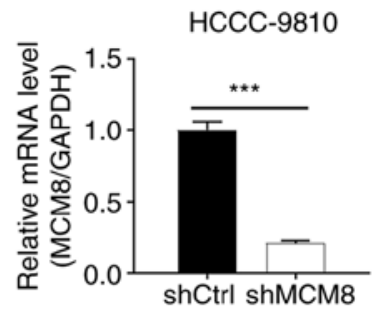

RBE

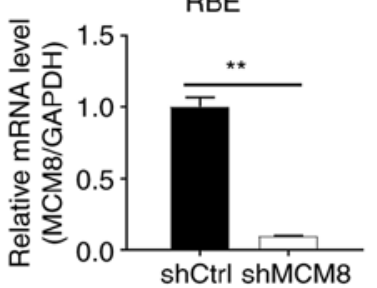

D

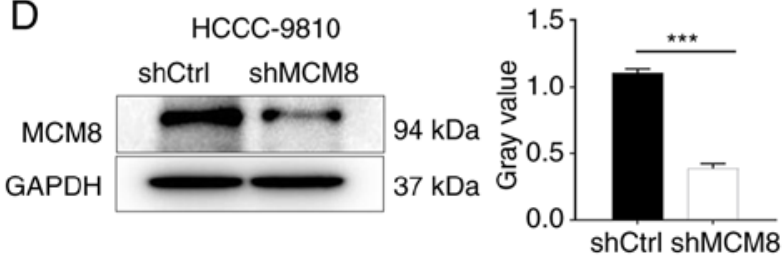

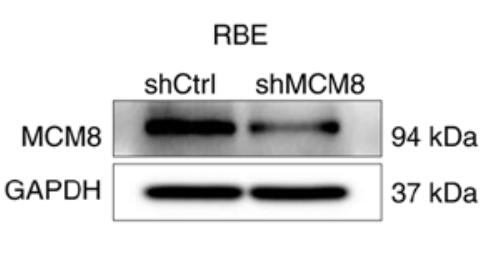

Figure 1. Expression level of MCM8 in CCA. (A) The expression level of MCM8 in paracancerous tissues and tumor tissues of CCA patients was determined by immunohistochemical staining. (B) The efficiency of lentivirus transduction of HCCC-9810 and RBE cells was evaluated by the expression of green fluorescent protein. (C and D) The expression of MCM8 in HCCC-9810 and RBE cells after the stable transduction of lentivirus shMCM8 was assessed by (C) quantitative PCR and (D) western blot analysis. The data are presented as the mean \pm SD $(\mathrm{n}=3)$. ${ }^{* *} \mathrm{P}<0.01$ and ${ }^{* * * *} \mathrm{P}<0.001$ compared with control shCtrl. MCM8, minichromosome maintenance 8 homologous recombination repair factor; CCA, cholangiocarcinoma; sh, short hairpin.

Statistical analysis. Experimental data were presented as the mean \pm standard deviation $(n=3)$ and statistical analyses were performed using SPSS 19.0 (IBM Corp.). The relationship between MCM8 expression and tumor characteristics in patients with CCA was analyzed using chi-square test or Fisher's exact test. Data produced using RT-qPCR was analyzed using $2^{-\Delta \Delta \mathrm{Cq}}$ method. The results of the MTT OD 490-nm value were obtained and wound-healing as well as Transwell assays were investigated using the paired two-tailed Student's t-test or one-way ANOVA followed by Bonferroni's post hoc test analysis. The relationship between MCM8 and clinicopathological features of CCA were analyzed using Mann-Whitney U and Pearson's correlation analysis. $\mathrm{P}<0.05$ was considered to indicate a statistically significant difference.

\section{Results}

Upregulation of MCM8 in CCA. The results of IHC staining revealed a lower signal intensity of MCM8 in paracancerous tissues when compared with CCA tissues, which indicated that MCM8 expression was significantly higher in CCA tissues compared with paracancerous tissues (Table I and Fig. 1A). Moreover, the relationship between MCM8 expression and tumor characteristics in patients with CCA was analyzed using chi-square test or Fisher's exact test. The results 
Table II. Relationship between MCM8 expression and tumor characteristics in patients with cholangiocarcinoma.

\begin{tabular}{|c|c|c|c|c|}
\hline \multirow[b]{2}{*}{ Features of the patients } & \multirow[b]{2}{*}{$\begin{array}{c}\text { No. of patients } \\
89\end{array}$} & \multicolumn{2}{|c|}{ MCM8 expression } & \multirow[b]{2}{*}{ P-value } \\
\hline & & $\begin{array}{c}\text { Low } \\
47\end{array}$ & $\begin{array}{l}\text { High } \\
42\end{array}$ & \\
\hline Age (years) & & & & 0.930 \\
\hline$\leq 57$ & 46 & 25 & 21 & \\
\hline$>57$ & 43 & 22 & 21 & \\
\hline Sex & & & & 0.736 \\
\hline Male & 46 & 23 & 23 & \\
\hline Female & 43 & 24 & 19 & \\
\hline Grade & & & & 0.443 \\
\hline I & 5 & 4 & 1 & \\
\hline II & 66 & 35 & 31 & \\
\hline III & 18 & 8 & 10 & \\
\hline $\mathrm{N}$ & & & & 0.698 \\
\hline No & 58 & 32 & 26 & \\
\hline $\mathrm{N} 1$ & 31 & 15 & 16 & \\
\hline $\mathrm{T}$ & & & & 0.338 \\
\hline $\mathrm{T} 1$ & 7 & 4 & 3 & \\
\hline $\mathrm{T} 2$ & 61 & 34 & 27 & \\
\hline $\mathrm{T} 3$ & 17 & 8 & 9 & \\
\hline $\mathrm{T} 4$ & 3 & 0 & 3 & \\
\hline Tumor size & & & & 0.639 \\
\hline$\leq 3 \mathrm{~cm}$ & 50 & 28 & 22 & \\
\hline$>3 \mathrm{~cm}$ & 39 & 19 & 20 & \\
\hline Stage & & & & 0.078 \\
\hline 1 & 8 & 4 & 4 & \\
\hline 2 & 40 & 27 & 13 & \\
\hline 3 & 27 & 11 & 16 & \\
\hline 4 & 14 & 5 & 9 & \\
\hline Lymphoid positive number & & & & 0.659 \\
\hline$\leq 0$ & 58 & 32 & 26 & \\
\hline$>0$ & 30 & 15 & 15 & \\
\hline
\end{tabular}

MCM8, minichromosome maintenance 8 homologous recombination repair factor.

Table III. Pearson correlation analysis of MCM8 expression and tumor characteristics in patients with cholangiocarcinoma.

\begin{tabular}{lcr}
\hline & \multicolumn{2}{c}{$\begin{array}{c}\text { Expression correlation } \\
\text { between MCM8 and stage }\end{array}$} \\
\cline { 2 - 3 } $\begin{array}{l}\text { Number of } \\
\text { samples }\end{array}$ & Pearson correlation coefficient & P-value \\
\hline 89 & 0.218 & 0.040
\end{tabular}

MCM8, minichromosome maintenance 8 homologous recombination repair factor.

indicated that there was no significant association between the expression level of MCM8 and the pathological stage of
CCA (Table II). In addition, Pearson's correlation analysis indicated that the higher the expression level of MCM8, the higher the pathological stage of CCA (Table III). Thus, investigating the expression levels of MCM8 may be clinically relevant in predicting the deterioration of CCA. Furthermore, CCA cell models with MCM8 knockdown were constructed to clarify the effects of MCM8 in CCA cells. The results of the RT-qPCR analysis revealed that the knockdown efficiency of MCM8 in the shMCM8-3 group was the highest compared with other groups (Fig. S1). In addition, a ratio of more than $80 \%$ of GFP was observed, indicating that the lentiviruses shCtrl and shMCM8 had successfully infected the CCA cells (Fig. 1B). The results of RT-qPCR demonstrated that the MCM8 expression was decreased by 79.3 and $90.7 \%$ in HCCC-9810 and RBE cells compared with the shCtrl group, respectively (Fig. 1C). The protein expression level 
A

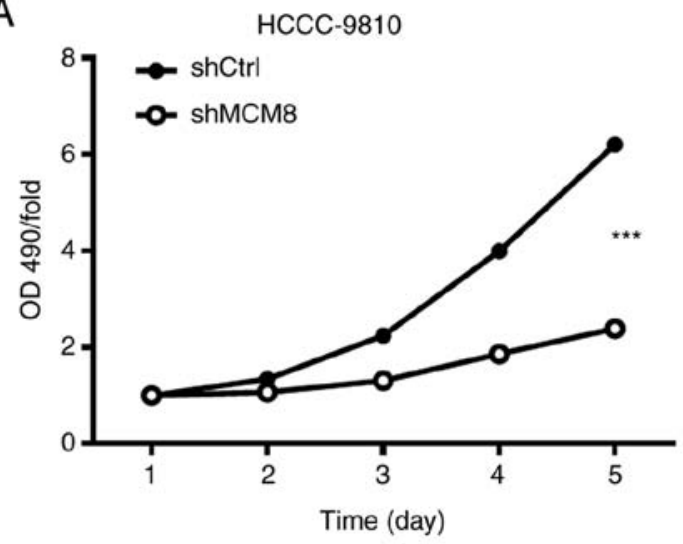

B
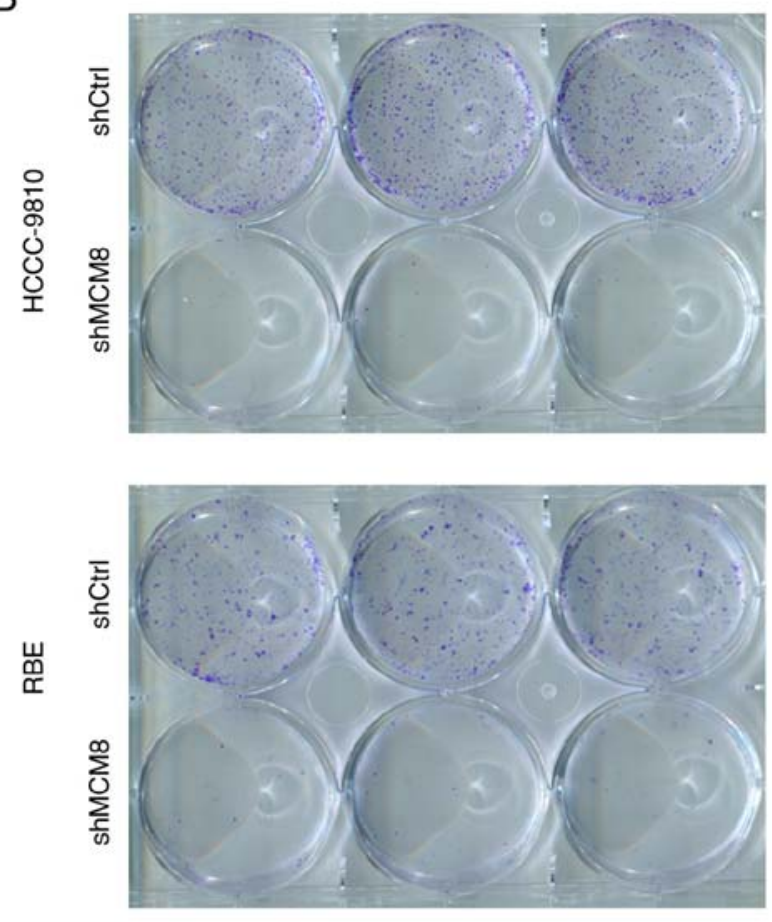
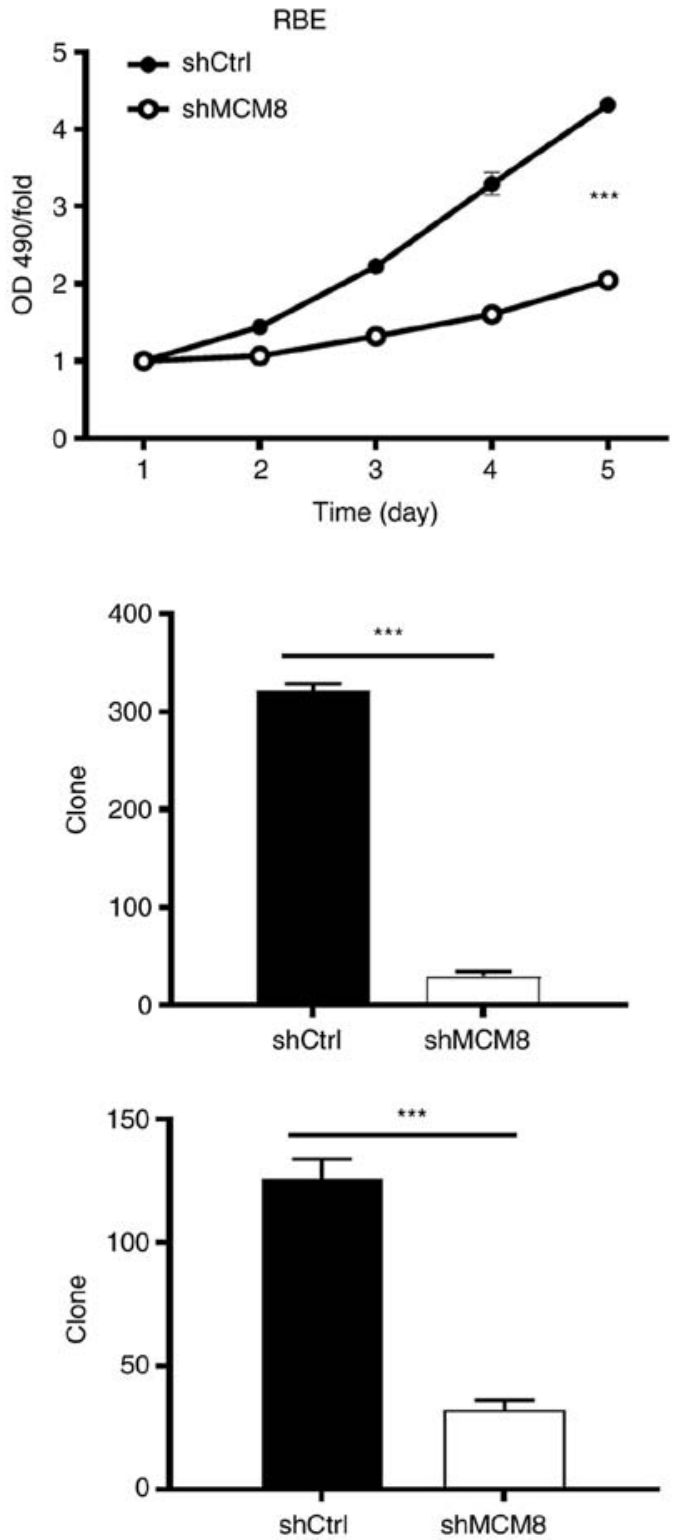

Figure 2. Decrease of MCM8 expression results in reduced proliferation of cholangiocarcinoma cells. (A and B) Lentivirus shMCM8-mediated HCCC-9810 and RBE cells were subjected to (A) MTT proliferation assay and (B) colony formation detection. The data are presented as the mean $\pm \mathrm{SD}(\mathrm{n}=3) .{ }^{* * * *} \mathrm{P}<0.001$ compared with shCtrl. MCM8, minichromosome maintenance 8 homologous recombination repair factor; sh, short hairpin.

of MCM8 was also decreased in HCCC-9810 and RBE cells infected with lentivirus shMCM8 (Fig. 1D). Consequently, the low shMCM8-mediated expression levels of MCM8 in HCCC-9810 and RBE cells were used for further functional analysis.

Reduced MCM8 expression suppresses HCCC-9810 and $R B E$ cell proliferation in vitro. As revealed in Fig. $2 \mathrm{~A}$, the effect of shCtrl and shMCM8 on the OD 490-nm values of HCCC-9810 and RBE cells was investigated. The results of the present study demonstrated that the level of cell proliferation was weakened in the shMCM8 group compared with the shCtrl group. Additionally, the colony formation assay in HCCC-9810 and RBE cells indicated that the shMCM8 group yielded smaller and fewer colonies compared with the control group (Fig. 2B). In conclusion, CCA cells with reduced MCM8 expression exhibited impaired proliferation.
Reduced MCM8 expression increases HCCC-9810 and RBE cell apoptosis in vitro. The level of cell apoptosis was assessed using flow cytometry. The results indicated that the decrease of MCM8 expression greatly enhanced the apoptotic levels of HCCC-9810 and RBE cells (Fig. 3A). Furthermore, expression levels of a number of apoptotic factors associated with apoptotic signaling pathways were analyzed in cells transfected with shCtrl and shMCM8. As revealed in the Fig. 3B, the expression levels of HSP27, IGF-1sR, survivin, sTNF-R1, sTNF-R2, TNF- $\alpha$, TNF- $\beta$ and XIAP were significantly downregulated in HCCC-9810 cells. The results of the present study revealed that MCM8 knockdown enhanced the apoptosis of CCA cells and led to the abnormal downregulation of apoptosis-related proteins.

Reduced MCM8 expression suppresses HCCC-9810 and RBE cell migration in vitro. The effect of MCM8 knockdown on 
A
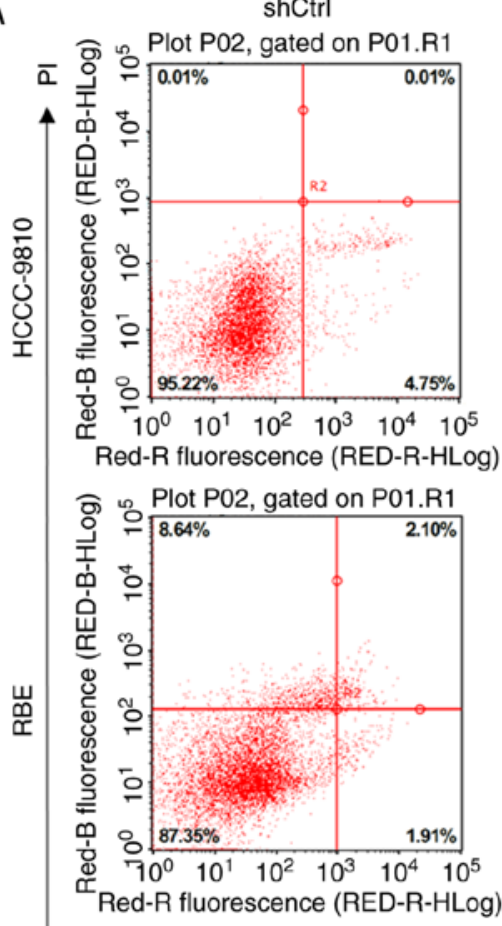

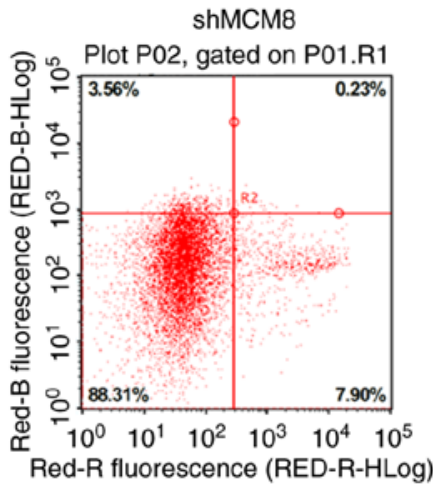

कิ

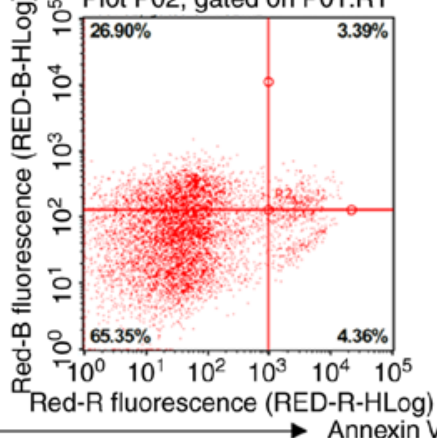

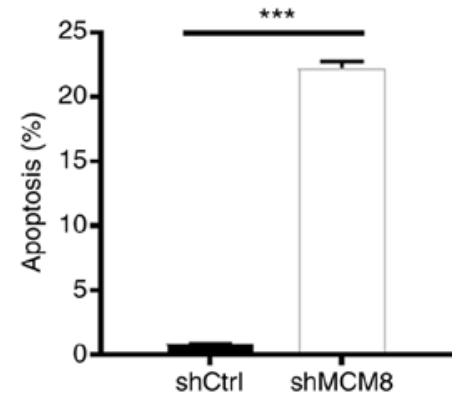

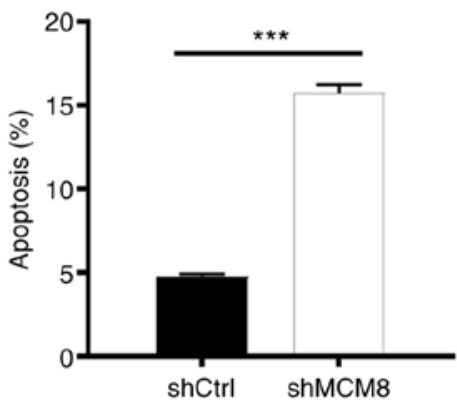

B
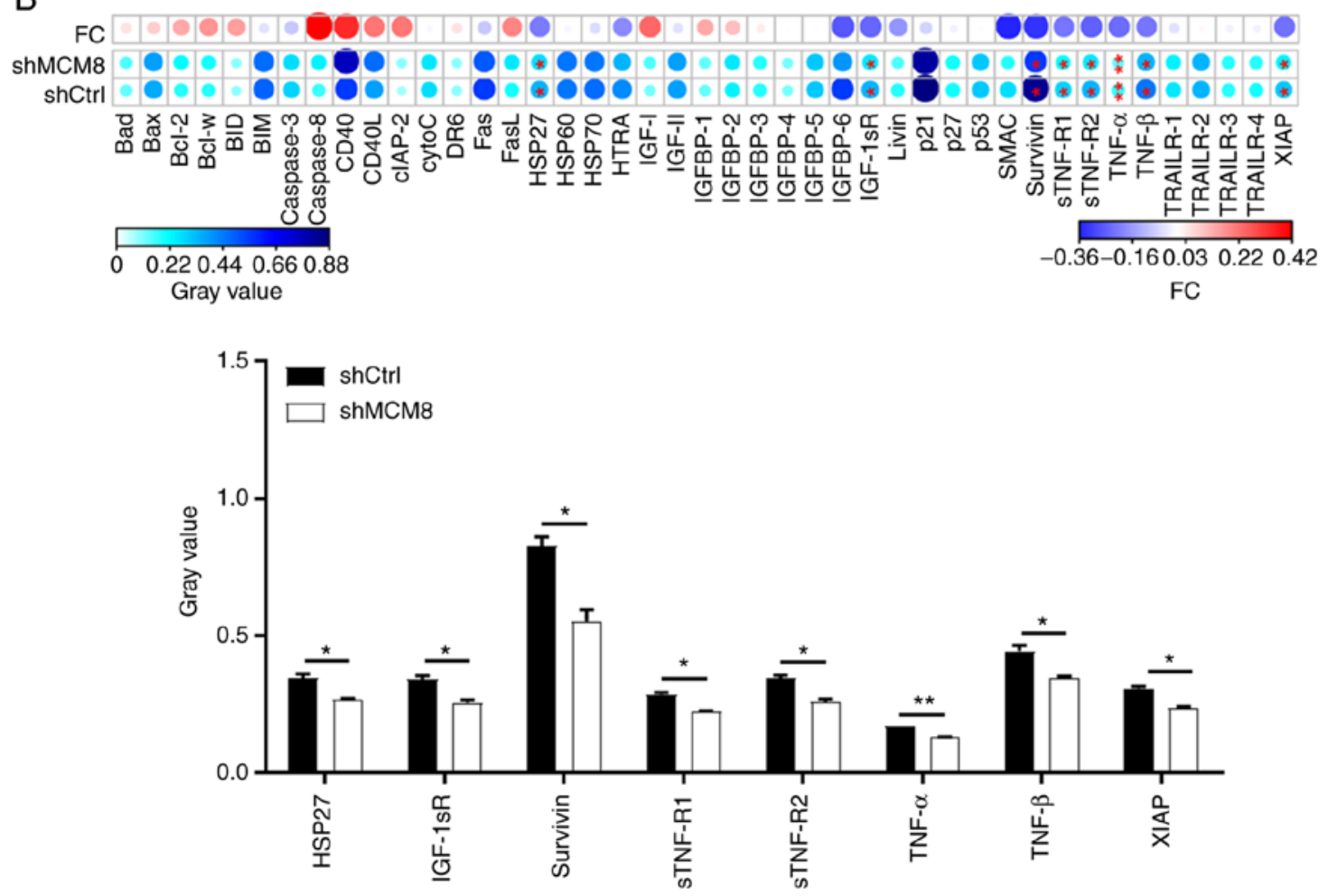

Figure 3. Decrease of MCM8 expression results in enhanced apoptosis of cholangiocarcinoma cells. (A) Lentivirus shMCM8-mediated HCCC-9810 and RBE cells subjected to apoptotic detection using flow cytometry based on Annexin V-APC staining. (B) Expression of apoptosis-related proteins was detected by the human apoptotic antibody array kit. The data are presented as the mean $\pm \mathrm{SD}(\mathrm{n}=3) .{ }^{*} \mathrm{P}<0.05,{ }^{* *} \mathrm{P}<0.01$, and ${ }^{* * *} \mathrm{P}<0.001$ compared with shCtrl. MCM8, minichromosome maintenance 8 homologous recombination repair factor; sh, short hairpin.

the migration ability of CCA cells was preliminarily evaluated using wound-healing and Transwell assays. Compared with the shCtrl group, the decreased expression of MCM8 resulted in a $16.9 \%$ reduction in the migration rate of HCCC-9810 cells within $24 \mathrm{~h}$. The migration rate of RBE cells at $0-48 \mathrm{~h}$ decreased by $26.7 \%$ (Fig. 4A). Additionally, the results of Transwell assay further indicated that the migration ability of HCCC-9810 and RBE cells in the MCM8-knockdown group was inhibited (Fig. 4B). In conclusion, knockdown of MCM8 inhibited the migration of CCA cells. To further investigate the effect of 
A

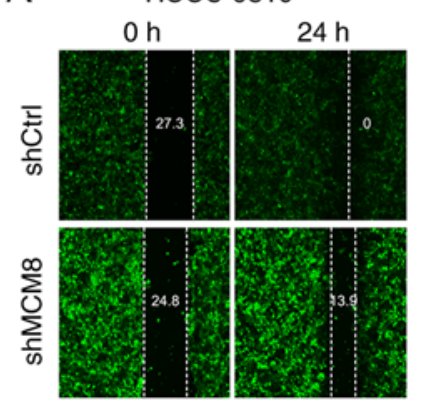

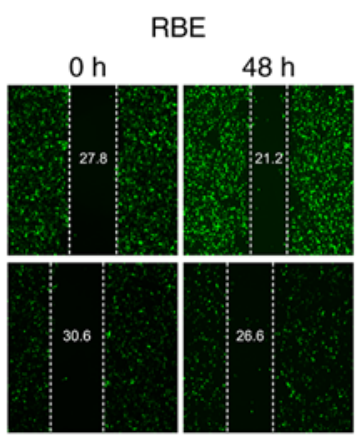

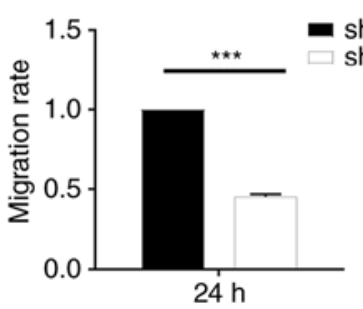

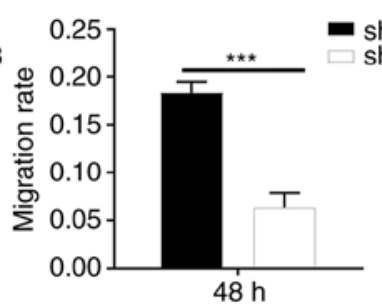

C

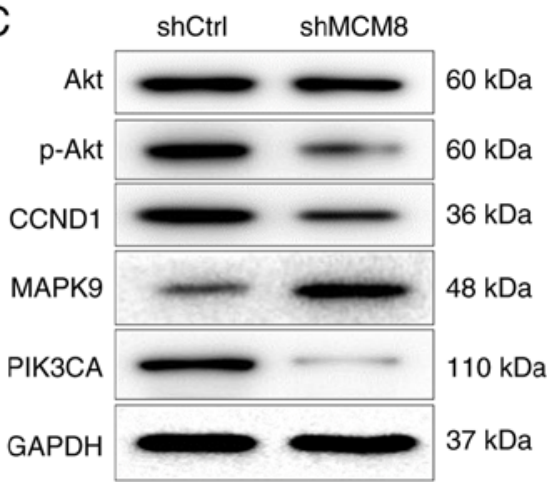

B
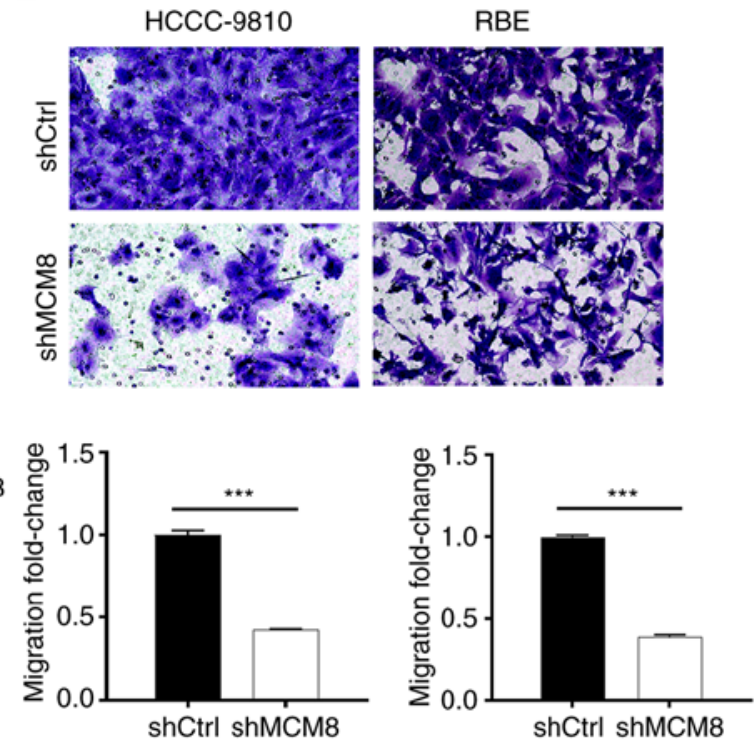

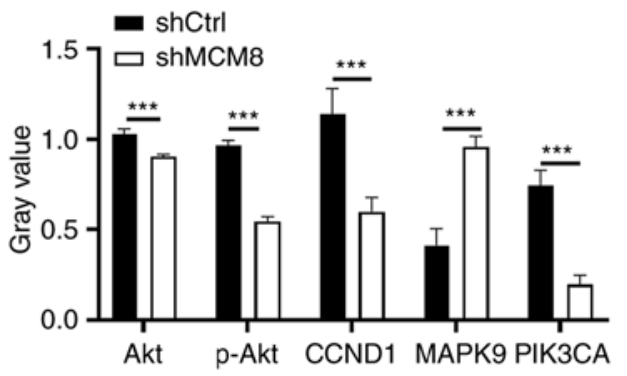

Figure 4. Decrease of MCM8 expression results in impeded migration of cholangiocarcinoma cells. (A and B) Lentivirus shMCM8-mediated HCCC-9810 and RBE cells subjected to (A) wound-healing (magnification, x200) and (B) Transwell assays (magnification, $\mathrm{x} 200$ ). (C) The expression of downstream signaling pathway proteins was analyzed by western blotting. The data are presented as the mean $\pm \mathrm{SD}(\mathrm{n}=3)$. ${ }^{* * *} \mathrm{P}<0.001$ compared with shCtrl. MCM8, minichromosome maintenance 8 homologous recombination repair factor; sh, short hairpin.

MCM8 on protein expression in downstream pathways, the expression levels of a number of proteins in well-established signaling pathways were detected in HCCC-9810 cells. The results of western blot analysis indicated that the expression level of MAPK9 was upregulated, while the expression levels of phosphorylated Akt, G1/S-specific cyclin D1 (CCND1) and PIK3 catalytic subunit alpha (CA) were downregulated in the shMCM8 group (Fig. 4C). Mechanistically, the knockdown of MCM8 led to abnormalities in the expression levels of proteins in well-established downstream pathways, such as PI3K/Akt, CCND1 and MAPK.

Reduced MCM8 expression impairs tumor generation in vivo. shMCM8 and shCtrl-mediated HuCCT1 cells were subcutaneously injected into nude mice to determine the effect of MCM8 knockdown in mice. Both groups of mice were anesthetized and images were subsequently captured using an imaging system. The results demonstrated that the intensity of bioluminescence in the shMCM8 group was significantly weaker compared with shCtrl group (Fig. 5A). The results indicated that tumor burden was reduced in mice with low MCM8 expression (Fig. 5B and C). Monitoring of the experimental mice for more than a month revealed that the largest tumor weighed $1.33 \mathrm{~g}$, with a volume of $1,399.91 \mathrm{~mm}^{3}$. In addition, mice with MCM8 knockdown developed tumors that were smaller in size. A maximum tumor diameter of $17.23 \mathrm{~mm}$ was observed in the shCtrl group and only $11.59 \mathrm{~mm}$ in the shMCM8 group (Fig. 5D). The IHC staining displayed in Fig. 5E further demonstrated that the expression of Ki67 was lower in the shMCM8 group compared with the control group. These results indicated that downregulation of MCM8 suppressed tumor formation in vivo.

\section{Discussion}

MCM8 is involved in the initiation and elongation during DNA replication $(13,17,18)$. The high growth stimulation of carcinogenesis leads to a high level of pressure on cancer cells to proliferate, compared with non-cancerous cells $(19,20)$. Furthermore, MCM8 plays an essential role in the repair process of replication stress. Thus, it was hypothesized that MCM8 may be associated with tumor progression. In addition, the results of a previous study revealed that the upregulation of MCM8 led to increased aggressiveness in a variety of human tumors (21). For example, elevated expression of MCM8 resulted in increased aggressiveness in 

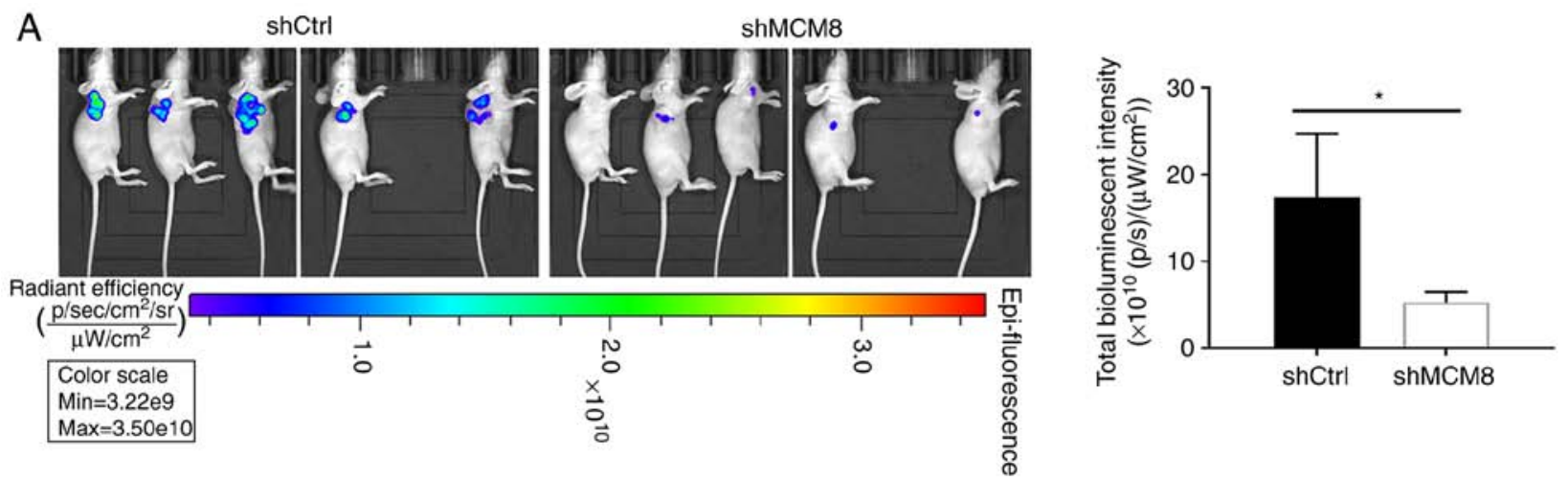

B

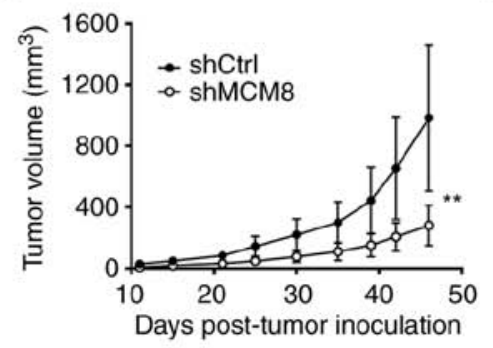

E

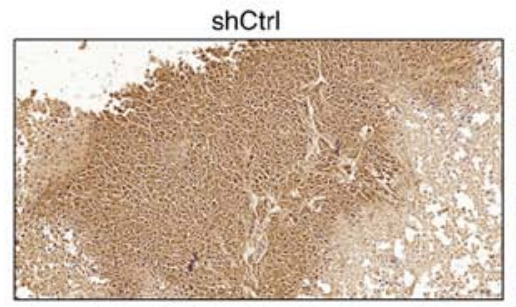

C

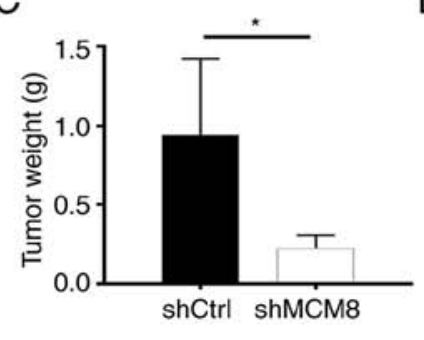

D

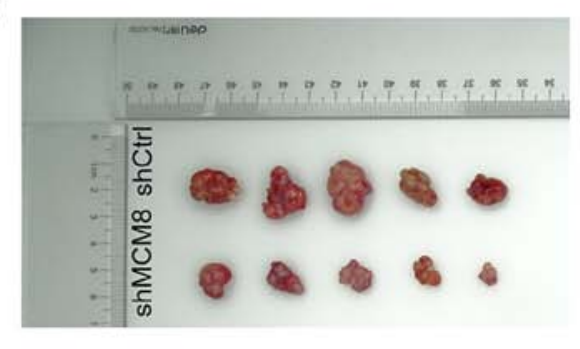

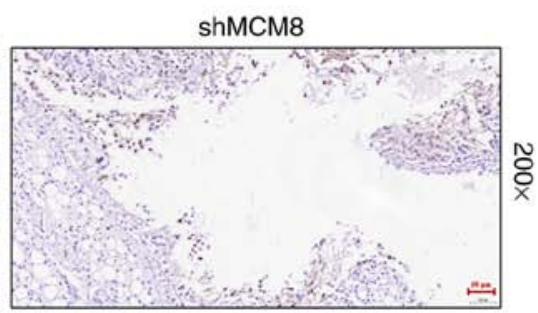

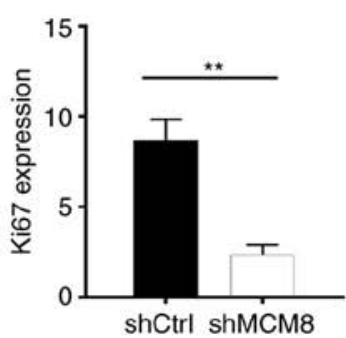

Figure 5. Decrease of MCM8 expression results in reduced cholangiocarcinoma tumorigenesis in mice. (A) The total tumor bioluminescence intensity of mice in the shCtrl group and the shMCM8 group. (B) Tumor volumes in the shCtrl group and shMCM8 groups were monitored continuously for 46 days. (C and D) Tumors in the shCtrl group and shMCM8 groups were removed, weighed and images were captured 46 days after cell injection. (E) The expression of Ki67 staining images in tumor tissues in the shCtrl group and the shMCM8 group. The data were presented as the mean $\pm \mathrm{SD}(\mathrm{n}=3)$. ${ }^{*} \mathrm{P}<0.05$ and ${ }^{* * *} \mathrm{P}<0.01$ compared with shCtrl. MCM8, minichromosome maintenance 8 homologous recombination repair factor; sh, short hairpin.

prostate cancer (21). MCM8 is also considered as an independent prognostic factor in patients with pancreatic cancer (22). However, the physiological functions and potential molecular mechanisms underlying MCM8 in CCA are yet to be fully elucidated.

In the present study, the overexpression of MCM8 in CCA was preliminarily identified using IHC staining. The biological function of MCM8 was investigated using a lentivirus-mediated CCA cell model. The loss-of-function assays revealed that the reduced expression of MCM8 resulted in reduced proliferation, induction of apoptosis and suppressed migration of CCA cells in vitro. Furthermore, decreased expression of MCM8 led to the decreased ability of tumor formation in mice. Thus, knockdown of MCM8 inhibited the malignant behavior of CCA, indicating that MCM8 exerted a role in the promotion of CCA.

Mechanistically, MCM8 knockdown contributed to the abnormal downregulation of apoptosis-related proteins such as survivin, XIAP, HSP27, IGF-1sR, sTNF-R1, sTNF-R2, TNF- $\alpha$ and TNF- $\beta$. Furthermore, MCM 8 knockdown induced apoptosis in CCA cells, which required a number of pro-apoptotic and anti-apoptotic factors. However, the exact mechanism underlying MCM8 regulation of CCA cell apoptosis is yet to be fully elucidated, and requires further discussion. In addition, Abnormalities in intracellular signaling pathways affected cell proliferation, survival, migration or invasion. Previous studies have demonstrated that PI3K/Akt, CCND1/CDK6 and MAPK signaling pathways serve key functions in CCA cells (23-26). For instance, Wang et al revealed that the PI3K/Akt signaling pathway played a critical role in mediating epithelial-mesenchymal transition and metastasis of CCA (27). Additionally, knockdown of CCND1 expression inhibited the proliferation and migration of CCA cells (28). Proline-rich homeodomain protein transcription factor is an oncogenic driver of CCA and is sensitive to CDK4/6 inhibitors (29). Zhang et al indicated that an abnormal p38/MAPK signaling pathway affected the proliferation of CCA cells (30). In conclusion, the PI3K/Akt, CCND1/CDK6 and MAPK signaling pathways played an important role in the progression of CCA cells. The present study demonstrated that the decrease of MCM8 expression inhibited the phosphorylation of Akt, downregulated the expression levels of CCND1, PIK3CA, and upregulated the expression of MAPK9. Therefore, it was hypothesized that MCM8 is involved in the progression of 
CCA cells through regulation of the PI3K/Akt, CCND1 and MAPK9 signaling pathways.

The present study, however, still has certain limitations. For instance, the clinical sample size that was used was limited. In addition, the present study only initially screened the changes of certain apoptosis-related proteins, and the exact mechanism of MCM8 affecting CCA apoptosis still requires a large number of studies for more in-depth insights.

The findings of the present study indicated that the high expression of MCM8 in CCA has the potential to be clinically significant in predicting disease progression. In addition, knockdown of MCM8 decreased proliferation and migration, and promoted apoptosis of CCA cells. In summary, MCM8 played a key role in the malignant progression of CCA, indicating that inhibition of MCM8 may prove to be of value as a molecular targeted therapy.

\section{Acknowledgements}

Not applicable.

\section{Funding}

The present study was supported by the Institutional Research Funding from The First Affiliated Hospital of Chengdu Medical College (grant no. CYFY-GQ20).

\section{Availability of data and materials}

The datasets used and/or analyzed during the current study are available from the corresponding author on reasonable request.

\section{Authors' contributions}

HDu designed the present study. HDe, LC, PY, JL and BL completed all in vitro experiments operation. YY, QW and XJ performed the animal assays. Data collection and statistical analysis were performed by HW. JH wrote the manuscript and it was reviewed by $\mathrm{HDu}$. JH and HDu confirm the authenticity of all the raw data. All authors read and approved the final manuscript.

\section{Ethics approval and consent to participate}

The experimental protocols and the human tissues used were approved (approval no. 20200305) by the Ethics Committee of Clinical Research of The First Affiliated Hospital of Chengdu Medical College (Chengdu, China). Mouse experiments were approved (approval no. 20190213) by the Ethics Committee of Chengdu Medical College, following the Guidelines and Procedures for Animal Care and Protection.

\section{Patient consent for publication}

Not applicable.

\section{Competing interests}

The authors declare that they have no competing interests.

\section{References}

1. Khan AS and Dageforde LA: Cholangiocarcinoma. Surg Clin North Am 99: 315-335, 2019.

2. Buckholz AP and Brown RS Jr: Cholangiocarcinoma: Diagnosis and management. Clin Liver Dis 24: 421-436, 2020.

3. Dondossola D, Ghidini M, Grossi F, Rossi G and Foschi D: Practical review for diagnosis and clinical management of perihilar cholangiocarcinoma. World J Gastroenterol 26: 3542-3561, 2020.

4. Banales JM, Marin JJ, Lamarca A, Rodrigues PM, Khan SA Roberts LR, Cardinale V, Carpino G, Andersen JB, Braconi C, et al: Cholangiocarcinoma 2020: The next horizon in mechanisms and management. Nat Rev Gastroenterol Hepatol 17: 557-588, 2020.

5. Rizvi S, Khan SA, Hallemeier CL, Kelley RK and Gores GJ: Cholangiocarcinoma-evolving concepts and therapeutic strategies. Nat Rev Clin Oncol 15: 95-111, 2018.

6. Labib PL, Goodchild G and Pereira SP: Molecular pathogenesis of cholangiocarcinoma. BMC Cancer 19: 185, 2019.

7. Zhang T, Chen G, Liu C, Zu L, Wang Q, Wang Y, Lv J, An Y, Dong L, Cheng H, et al: A phase I Study comparing the pharmacokinetics, safety, and immunogenicity of proposed biosimilar GB242 and reference infliximab in healthy subjects. BioDrugs 33: 93-100, 2019.

8. Zhai Y, Li N, Jiang H, Huang X, Gao N and Tye BK: Unique roles of the non-identical MCM subunits in DNA replication licensing. Mol Cell 67: 168-179, 2017.

9. Zhong X, Chen X, Guan X, Zhang H, Ma Y, Zhang S, Wang E, Zhang L and Han Y: Overexpression of G9a and MCM7 in oesophageal squamous cell carcinoma is associated with poor prognosis. Histopathology 66: 192-200, 2015.

10. Das M, Prasad SB, Yadav SS, Govardhan HB, Pandey LK, Singh S, Pradhan S and Narayan G: Over expression of minichromosome maintenance genes is clinically correlated to cervical carcinogenesis. PLoS One 8: e69607, 2013.

11. Giaginis C, Giagini A, Tsourouflis G, Gatzidou E, Agapitos E, Kouraklis G and Theocharis S: MCM-2 and MCM-5 expression in gastric adenocarcinoma: Clinical significance and comparison with Ki-67 proliferative marker. Dig Dis Sci 56: 777-785, 2011.

12. Cobanoglu U, Mungan S, Gundogdu C, Ersoz S, Ozoran Y and Aydin F: The expression of MCM-2 in invasive breast carcinoma: A stereologic approach. Bratisl Lek Listy 111: 45-49, 2010.

13. Gozuacik D, Chami M, Lagorce D, Faivre J, Murakami Y, Poch O, Biermann E, Knippers R, Bréchot C and Paterlini-Bréchot P: Identification and functional characterization of a new member of the human $\mathrm{Mcm}$ protein family: $\mathrm{hMcm} 8$. Nucleic Acids Res 31: 570-579, 2003.

14. Liu Z, Li J, Chen J, Shan Q, Dai H, Xie H, Zhou L, Xu X and Zheng S: MCM family in HCC: MCM6 indicates adverse tumor features and poor outcomes and promotes $\mathrm{S} / \mathrm{G} 2$ cell cycle progression. BMC Cancer 18: 200, 2018.

15. Haonon O, Rucksaken R, Pinlaor P, Pairojkul C, Chamgramol Y, Intuyod $\mathrm{K}$, Onsurathum $\mathrm{S}$, Khuntikeo $\mathrm{N}$ and Pinlaor $\mathrm{S}$ : Upregulation of 14-3-3 eta in chronic liver fluke infection is a potential diagnostic marker of cholangiocarcinoma. Proteomics Clin Appl 10: 248-256, 2016.

16. Livak KJ and Schmittgen TD: Analysis of relative gene expression data using real-time quantitative PCR and the 2(-Delta Delta C(T)) method. Methods 25: 402-408, 2001.

17. Maiorano D, Cuvier O, Danis E and Méchali M: MCM8 is an MCM2-7-related protein that functions as a DNA helicase during replication elongation and not initiation. Cell 120: 315-328, 2005.

18. Volkening $M$ and Hoffmann I: Involvement of human MCM8 in prereplication complex assembly by recruiting hede6 to chromatin. Mol Cell Biol 25: 1560-1568, 2005.

19. Kotsantis P, Silva LM, Irmscher S, Jones RM, Folkes L, Gromak $\mathrm{N}$ and Petermann E: Increased global transcription activity as a mechanism of replication stress in cancer. Nat Commun 7: 13087, 2016.

20. Hills SA and Diffley JF: DNA replication and oncogene-induced replicative stress. Curr Biol 24: R435-R444, 2014.

21. He DM, Ren BG, Liu S, Tan LZ, Cieply K, Tseng G, Yu YP and Luo JH: Oncogenic activity of amplified miniature chromosome maintenance 8 in human malignancies. Oncogene 36: 3629-3639, 2017.

22. Peng YP, Zhu Y, Yin LD, Zhang JJ, Guo S, Fu Y, Miao Y and Wei JS: The expression and prognostic roles of MCMs in pancreatic cancer. PLoS One 11: e0164150, 2016. 
23. Song X, Liu X, Wang H, Wang J, Qiao Y, Cigliano A, Utpatel K, Ribback S, Pilo MG, Serra M, et al: Combined CDK4/6 and Pan-mTOR inhibition is synergistic against intrahepatic cholangiocarcinoma. Clin Cancer Res 25: 403-413, 2019.

24. Samukawa E, Fujihara S, Oura K, Iwama H, Yamana Y, Tadokoro T, Chiyo T, Kobayashi K, Morishita A, Nakahara M, et al: Angiotensin receptor blocker telmisartan inhibits cell proliferation and tumor growth of cholangiocarcinoma through cell cycle arrest. Int J Oncol 51: 1674-1684, 2017.

25. Zhang Y, Ji G, Han S, Shao Z, Lu Z, Huo L, Zhang J, Yang R, Feng Q, Shen H, et al: Tip60 Suppresses cholangiocarcinoma proliferation and metastasis via PI3k-AKT. Cell Physiol Biochem 50: 612-628, 2018.

26. Peng R, Zhang PF, Zhang C, Huang XY, Ding YB, Deng B, Bai DS and Xu YP: Elevated TRIM44 promotes intrahepatic cholangiocarcinoma progression by inducing cell EMT via MAPK signaling. Cancer Med 7: 796-808, 2018.

27. Wang Y, Liang Y, Yang G, Lan Y, Han J, Wang J, Yin D, Song R, Zheng T,Zhang S, et al: Tetraspanin 1 promotes epithelial-to-mesenchymal transition and metastasis of cholangiocarcinoma via PI3K/AKT signaling. J Exp Clin Cancer Res 37: 300, 2018.
28. Chang W, Wang Y, Li W, Shi L and Geng Z: MicroRNA-551b-3p inhibits tumour growth of human cholangiocarcinoma by targeting Cyclin D1. J Cell Mol Med 23: 4945-4954, 2019.

29. Kitchen P, Lee KY, Clark D, Lau N, Lertsuwan J, Sawasdichai A, Satayavivad J, Oltean S, Afford S, Gaston K and Jayaraman PS: A runaway PRH/HHEX-notch3-positive feedback loop drives cholangiocarcinoma and determines response to CDK4/6 inhibition. Cancer Res 80: 757-770, 2020.

30. Zhang MX, Gan W, Jing CY, Zheng SS, Yi Y, Zhang J, Xu X, Lin JJ, Zhang BH and Qiu SJ: S100A11 promotes cell proliferation via P38/MAPK signaling pathway in intrahepatic cholangiocarcinoma. Mol Carcinog 58: 19-30, 2019. Attribution-NonCommercial-NoDerivatives 4.0 International (CC BY-NC-ND 4.0) License. 